\title{
DISTRIBUCIÓN ESPACIAL DE LA HUMEDAD Y SU RELACIÓN CON LA TEXTURA EN UN SUELO
}

\begin{abstract}
Kenneth Largaespada ${ }^{1 / *}$, Carlos Henríquez*
Palabras clave: Reflectómetro de dominio tiempo (TDR); propiedades físicas del suelo; geoestadística; humedad volumétrica del suelo.

Keywords: Time domain reflectrometer (TDR); physical soil properties; geostatistics; volumetric soil moisture.
\end{abstract}

Recibido: $13 / 04 / 15$

\section{RESUMEN}

En Finca Esmeralda (Limón, Costa Rica), sembrada de banano cv. Valery, se analizó la distribución espacial de la humedad del suelo y su relación con algunas de sus propiedades físicas, para determinar la variabilidad entre el método tradicional y el uso de TDR (Time Domain Reflectrometer) en la determinación de la humedad del suelo. Se muestreó en forma de cuadrícula, con 36 puntos de medición georeferenciados con GPS a 2 profundidades del suelo. En cada punto se midió el agua volumétrica del suelo con 3 diferentes equipos de TDR (300, MP y MT) y se comparó con el método tradicional de determinación de humedad volumétrica (MHV). También se recolectaron muestras de suelo para análisis de textura; con estos datos se hizo un análisis geoestadísitico y se elaboraron de los mapas correspondientes. Los suelos de texturas Franco a Franco arcillosas, mostraron variabilidad entre las determinaciones con TDR y estas en relación con el MHV, independientemente de la profundidad. A nivel superficial $(0-30 \mathrm{~cm})$, la correlación más alta fue entre los valores del MHV y el TDR-300 ( $r=0,69)$, seguida del TDRMT $(r=0,63)$ y finalmente del TDR-MP $(r=0,59)$.

$1 \quad$ Autor para correspondencia. Correo electrónico: kenneth.largaespada@ucr.ac.cr

Aceptado: 06/07/15

\section{ABSTRACT}

Spatial distribution of moisture and its relation with soil texture. At Esmeralda Farm (Guácimo, Limón Province, C.R.), planted to banana cv. Valery, the spatial distribution of soil humidity, and its relationship to some physical properties, were analyzed to determine the variability between the traditional method and use of TDR (Time Domain Reflectrometer) in the determination of soil humidity. Sampling was done in a quadricuar pattern, with 36 measurement points georeferenced by GPS at 2 soil depths. At each point the volumetric soil water was measured with 3 different TDR equipments (300, MP and MT), and compared with the traditional method of volumetric humidity (VHM) determination. Soil samples were also collected, for texture analysis; with these data, a geostatistical analysis was performed and the corresponding maps were drafted. The soils, of Loam to clayey Loam texture, showed variability between TDR and these determinations regarding the MHV, regardless of depth. On the surface, the highest correlation was found between the values of MHV and TDR-300 ( $r=0.69$ ), followed by TDRMT ( $r=0.63)$ and finally the TDR-MP $(r=0.59)$.

Centro de Investigaciones Agronómicas y Sede del Atlántico de la Universidad de Costa Rica. San José, Costa Rica. 
En la capa subsuperficial (30 a $60 \mathrm{~cm}$ ), se encontró igualmente una relación positiva, pero menor, entre los valores del MHV con respecto al TDR300 y TRD-MP (0,47 y de 0,38 , respectivamente); no se encontró relación con la utilización del TDR-MT. En términos del mapa de humedad de campo, hubo buena representación entre los métodos, que en general fueron eficaces para representar la variación espacial de la humedad del suelo.
At 30 to $60 \mathrm{~cm}$ depth, a positive but lower ratio values was found compared MHV with TRD300 and TDR-MP (0.47 and 0.38, respectively); no relationship was found with TDR-MT at this depth. In terms of field moisture map, a good representation between methods was found and it can be said that this method was effective in representing the spatial variation of soil moisture.

\section{INTRODUCCIÓN}

El contenido de agua en el suelo es una de las principales limitantes para el crecimiento de las plantas. Tanto su exceso como su carencia, provocan un efecto que va en detrimento del rendimiento de los cultivos, por lo que su estimación y la variación de su contenido en el campo en forma rápida, es uno de los aspectos prioritarios para el manejo adecuado de este importante recurso en los agroecosistemas.

Se sabe que el contenido de agua en el suelo es fundamental para todos los procesos de crecimiento de las plantas, en particular en su nutrición, debido a que funciona como medio de transporte de los elementos a través de un mecanismo de flujo de masa lo cual incrementa el crecimiento y el rendimiento de los cultivos (Gavande 1973, Porta et ál. 2003, Torrán 2007).

Debido a lo anterior, la capacidad de retención de la humedad del suelo, viene a ser un aspecto fundamental para el desarrollo y crecimiento de las plantas, dicha capacidad va estar influenciada directamente por las propiedades y procesos físicos, químicos y biológicos del suelo por lo que su impacto sobre las plantas es indudable (Porta et ál. 2003). Asimismo es necesario, relacionar el contenido de humedad con otras variables del suelo como son textura y densidad aparente, las cuales como es de esperar presentan una variabilidad espacial importante en el campo o paisaje agrícola. El efecto de la textura sobre el agua del suelo se puede ver manifestado tanto en su almacenamiento como en su movimiento, y depende de las proporciones de arcilla o bien arenas, según sea el caso (Porta et ál. 2003, Henríquez y Cabalceta 2012). Esto hace que las metodologías de determinación de agua en el suelo, sean de mucha importancia, particularmente las mediciones que se realizan in situ las cuales permiten tomar decisiones en forma expedita, lo que evita cumplir con el tiempo del método tradicional, el cual es destructivo, lento (mínimo 2 días) y no permite repetir la estimación en un mismo punto. No obstante son exactos, precisos y baratos (Muñoz y Ritter 2005, Radulovich 2009). Los métodos de determinación de la humedad en el suelo a nivel de campo han sido mejorados sustancialmente, sin embargo a pesar de que existen en el mercado varios tipos de instrumentos, estos requieren de una calibración previa para poder ser usados en una condición particular. Una forma de estudiar esta variabilidad espacial es a través de la aplicación de la geoestadística, la cual permite caracterizar esta variación e integrar al mismo tiempo otras variables, que permiten llevar a cabo algunas relaciones entre las mismas. Ello permite sin duda optimizar la toma de decisiones con respecto al riego o el drenaje si es del caso, que son las prácticas agrícolas más importantes en relación con el agua de suelo. Con este estudio se 
pretendió hacer una integración de herramientas y procedimientos para estudiar la variación de la humedad del suelo en el paisaje agrícola.

\section{MATERIALES Y MÉTODOS}

\section{Lugar del ensayo}

El área de estudio se localizó en Finca Esmeralda la cual está dedicada al cultivo del banano, cultivar "Valery", propiedad de la empresa Chiquita Brands; se ubica en Guácimo, provincia de Limón, Costa Rica (Figura 1). El lugar tiene una temperatura y precipitación anual promedio de $27^{\circ} \mathrm{C}$ y $3970 \mathrm{~mm}$ respectivamente. El suelo clasificado como Typic Hapludands, presentó una topografía plana. Para llevar a cabo el estudio, se procedió a seleccionar un área de $21780 \mathrm{~m}^{2}$ la cual para efectos del ensayo se extendió a lo largo de lo que en el cultivo del banano se denomina como "cable", el cual estaba posicionado en el centro del área. Así mismo se encontraba delimitada por drenajes secundarios en los 2 bordes, por un camino al frente del área de estudio y un drenaje primario al final del "cable". En el área seleccionada se ubicaron 36 puntos distribuidos a lo largo del "cable" (se consideraron 2 puntos a ambos lados del mismo), los cuales se georeferenciaron con GPS (Global Positioning System) que conformaron de esta forma, una cuadrícula irregular (Figura 1). Los puntos seleccionados se ubicaron siempre frente de una planta de banano a una distancia de $40 \mathrm{~cm}$ de la misma, luego se procedió a hacer determinaciones que se realizaron a 2 profundidades: una superficial (0-30 $\mathrm{cm})$ y la otra a $30 \mathrm{~cm}$. En cada punto y a las 2 profundidades se evaluaron las variables que se describen a continuación.

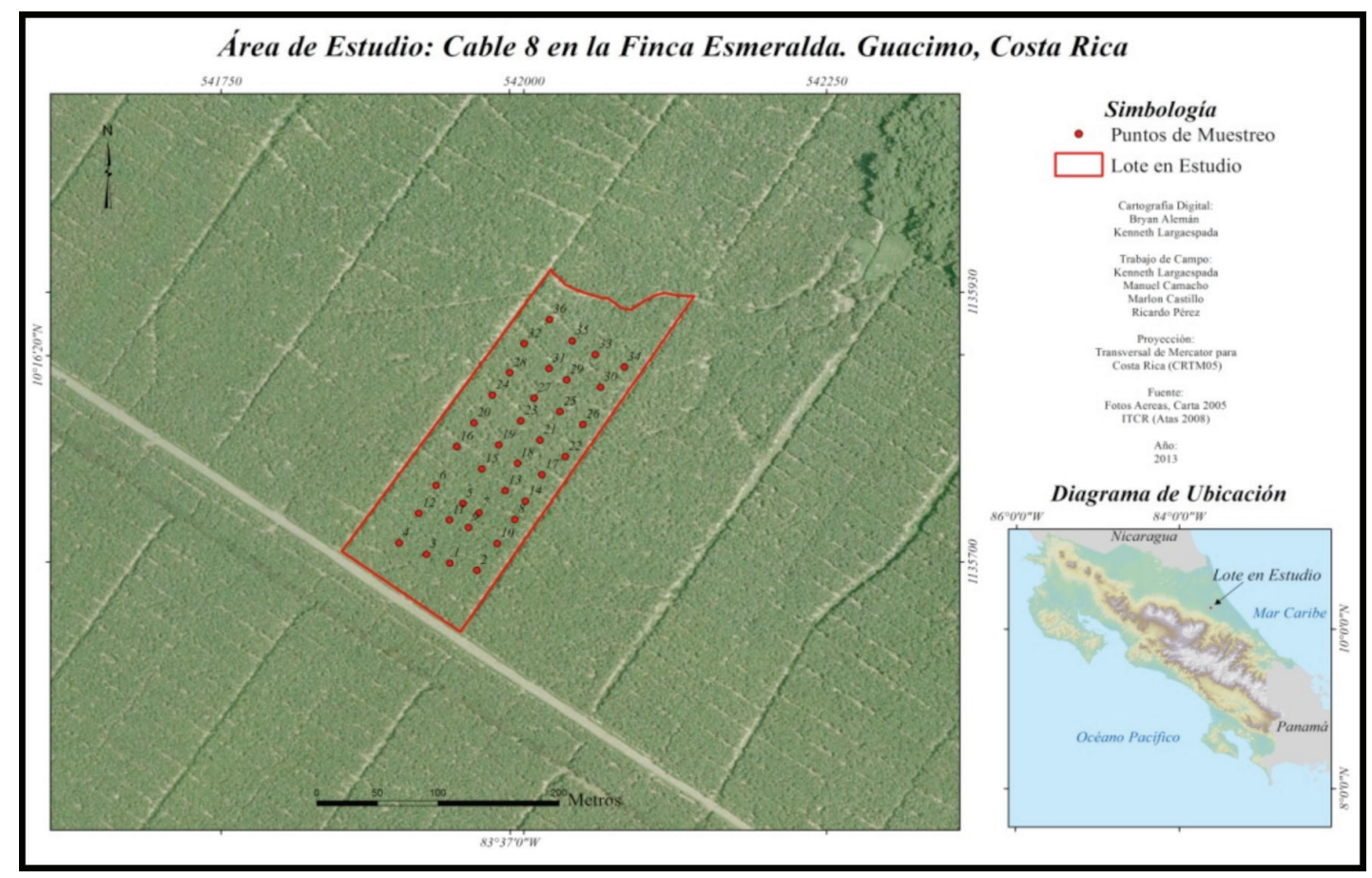

Fig. 1. Puntos de muestreo y ubicación geográfica del lugar del estudio ubicado en el "cable 8" en finca Esmeralda, Guácimo. 


\section{Determinación de la humedad por medio de Time Domain Reflectrometer (TDR)}

Las evaluaciones fueron realizadas bajo las mismas condiciones climáticas. Determinaciones de la humedad del suelo en condiciones de campo y recolección de muestras, se determinó la humedad in situ, en cada uno de los puntos de la cuadrícula se procedió a realizar diferentes determinaciones. Una de ellas fue la medición directa con 3 tipos diferentes de TDR (Time Domain Reflectometer), a 2 profundidades de suelo, (0 a $30 \mathrm{~cm}$ y de 30 a $60 \mathrm{~cm}$ ) en cada una de ellas se realizaron 4 determinaciones con los diferentes instrumentos. Los aparatos TDR utilizados para realizar las mediciones fueron: TDR Opti Science (TDR-300) que presenta un tipo de sonda de 2 varillas, con una longitud de 7,5 cm, TDR Soil Moisture MiniTrase (TDR-MT) con un tipo de sonda de 2 varillas una longitud de 15 $\mathrm{cm}$, TDR ICT (TDR- MP) con un tipo de sonda de 4 varillas y una longitud de $6 \mathrm{~cm}$. En este caso la diferencia entre los instrumentos fue el tipo de sonda y la longitud de la misma (Figura 2).

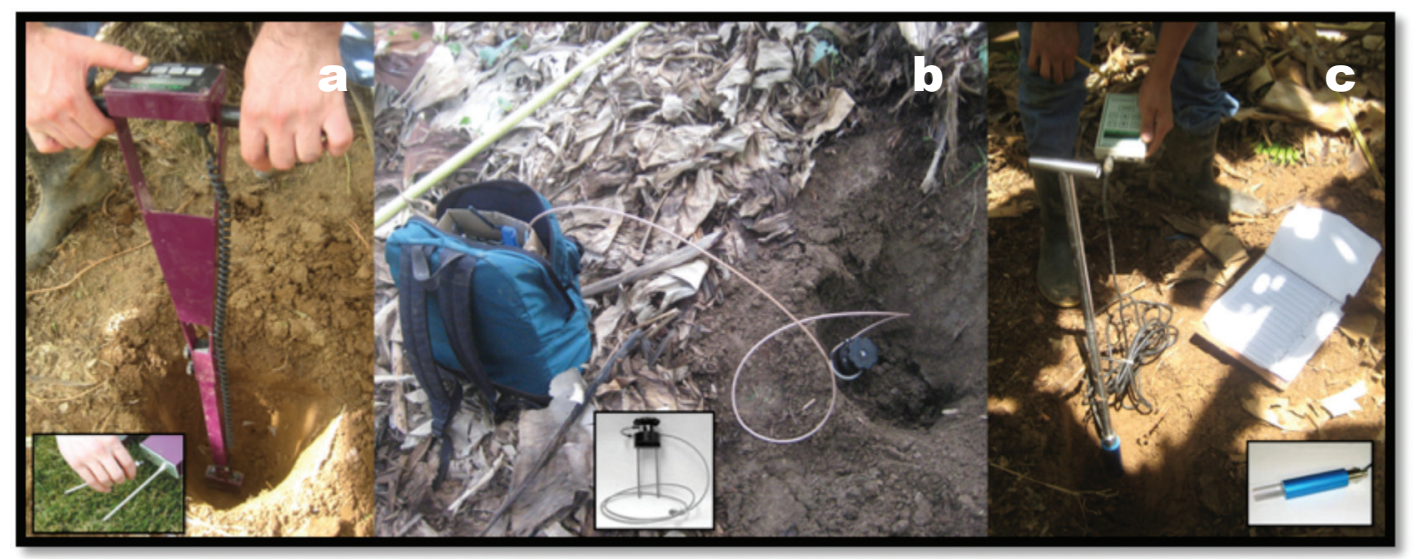

Fig. 2. TDR utilizados para determinación del contenido de humedad volumétrica in situ, a) Opti Science, b)Soil Moisture MiniTrase c) ICT.

Para hacer cada determinación, se procedió a limpiar la superficie con el fin de asegurarse de hacer las determinaciones sobre el suelo y no sobre alguna piedra o material extraño esto mismo se aplicó para las otras variables del estudio y tanto en la determinación a nivel superficial como a los $30 \mathrm{~cm}$ de profundidad. Para esta última se procedió a escarbar con una pala y medir con una cinta métrica hasta alcanzar la profundidad deseada de $30 \mathrm{~cm}$. Una vez lista la superficie de determinación, se introdujo suavemente en el suelo las sondas de cada TDR y se realizaron las mediciones respectivas (Topp y Ferré 2002).

\section{Determinación de la humedad método tradicional}

Para la humedad volumétrica tradicional, se utilizaron cilindros metálicos de acero de $146,6 \mathrm{~cm}^{3}$ de volumen $(5,4 \mathrm{~cm}$ de diámetro y 6,4 $\mathrm{cm}$ de altura), los cuales se introdujeron en el suelo con golpes similares en fuerza y frecuencia (a las 2 profundidades y a lo largo de los 36 puntos de estudio). Una vez lleno el cilindro, se sacaron con ayuda de una pala, y se limpiaron cuidadosamente para eliminar los sobrantes de suelo de los bordes del cilindro con ayuda de un cuchillo; las muestras fueron guardadas en 
papel aluminio y bolsas plásticas cerradas herméticamente para su transporte al laboratorio. Se pesaron con la humedad de campo y se colocaron en una estufa a $110^{\circ} \mathrm{C}$ por 48 horas, a partir de las metodologías descritas por Radulovich (2009) y Henríquez y Cabalceta (2012).

\section{Determinación de la textura del suelo}

Para la textura, se recolectaron muestras a las 2 profundidades determinadas para el estudio y a lo largo de los 36 puntos de muestreo se introdujo en bolsas plásticas; una vez el laboratorio se utilizó la metodología sugerida por Forsythe (1985), con la variación que se trabajo con muestra húmeda previamente tamizada por una malla de $2 \mathrm{~mm}$, con la ejecución de lecturas a los 40 segundos y posteriormente a las 2 horas, para determinar el nombre textural de cada punto y profundidad, según el sistema USDA.

\section{Variación espacial de la humedad del suelo en el lote de estudio}

A los datos obtenidos a partir de las diferentes variables evaluadas en las 2 profundidades y en correspondencia a su posición geográfica tomada con el GPS, fueron sometidos al análisis geoestadístico mediante la elaboración de semivariogramas para lo cual se utilizó el software GS+ V9 (GeoStatistics for the Environmental Sciences). Luego de determinar el modelo de regresión más idóneo (modelo de mejor ajuste), se elaboraron los mapas correspondientes de variación espacial de la humedad del suelo en el área de estudio. Con los parámetros geoestadísticos obtenidos, se elaboraron los mapas con el programa ArcGis 10.1 de ESRI. También se llevaron a cabo análisis estadísticos con la " $t$ de Student" y correlaciones simples de Pearson entre las diferentes metodologías de determinación y entre las variables, con el fin de encontrar algún tipo de concordancia con la variación de cada una de las variables evaluadas. Además de un análisis de correspondencia entre las interpolaciones realizadas del contenido de humedad para cada uno de los métodos.

\section{RESULTADOS Y DISCUSIÓN}

\section{Determinación de la humedad volumétrica}

Con los resultados de este estudio se encontró un nivel en el contenido de agua en el suelo diferencial tanto en las 2 profundidades (0-30 cm y 30-60 cm), como en los diferentes métodos de determinación (Cuadro 1). Estas diferencias de los TDR con respecto al método tradicional, podrían deberse entre otras cosas, al tipo de sonda de cada instrumento, que incluye el largo de la misma, el área de exploración y volumen de determinación de la humedad en el suelo. Esto concuerda con Martínez y Ceballo (2001) y Sánchez et ál. (1999), quienes mencionan que el tipo de sonda y el diseño de la misma, pueden presentar variaciones principalmente por aspectos como tipo de cable, conexión con la sonda, número de varillas, su disposición y dimensiones (longitud, diámetro y separación), así como características influenciadas en la forma de la señal del TDR y características en el voltaje entre otros. Pese a ello, es esperable que estas diferencias entre métodos sean mínimas. 
Cuadro 1. Parámetros estadísticos del porcentaje de humedad volumétrica medido con el método tradicional y 3 TDR a 2 profundidades del suelo en el "cable 8" en Finca Esmeralda, Guácimo, Costa Rica.

\begin{tabular}{lccccc}
\hline $\begin{array}{l}\text { Variable } \\
(\% \mathrm{HV})\end{array}$ & $\begin{array}{c}\text { Prof. } \\
(\mathrm{cm})\end{array}$ & Promedio & Máximo & Mínimo & Desviación estándar \\
\hline \multirow{2}{*}{ Método tradicional } & 0 a 30 & 46,02 & 61,44 & 34,63 & 6,5 \\
& 30 a 60 & 45,08 & 52,59 & 38,05 & 3,84 \\
\hline \multirow{2}{*}{ TDR-MP } & 0 a 30 & 40,89 & 46,25 & 33,2 & 3,43 \\
& 30 a 60 & 42,23 & 45,93 & 34,8 & 2,34 \\
\hline \multirow{2}{*}{ TDR-300 } & 0 a 30 & 62,48 & 78,77 & 41,9 & 10,16 \\
\hline \multirow{2}{*}{ TDR-MT } & 30 a 60 & 56,05 & 67,7 & 42,53 & 5,8 \\
\hline
\end{tabular}

Nota TDR Mini Trase con un N de 16 datos, dado que no se pudieron realizar las 36 mediciones como se explicara más adelante.

Al correlacionar las variables de distribución espacial de la humedad del suelo determinadas en este estudio, se encontró que a nivel superficial $(0-30 \mathrm{~cm})$ la correlación más alta fue entre los valores del método tradicional y el TDR-300, los cuales correlacionaron positivamente con un coeficiente de correlación (r) de 0,69 y en forma altamente significativa, datos que se presentan en el Cuadro 2; por otro lado el método tradicional y el TDR-MP también correlacionaron positivamente ( $\mathrm{r}$ de 0,59 y altamente significativa), al igual que el método tradicional y el TDR-MT ( $\mathrm{r}$ de 0,63 y altamente significativa).

En la capa subsuperficial $(30$ a $60 \mathrm{~cm}$ ), se encontró igualmente una relación positiva y altamente significativa entre los valores del método tradicional y el TDR-MP, así como de esta con el TRD-300 de $\mathrm{r}$ igual a 0,38 y de 0,47, altamente significativas respectivamente. Extrañamente, al relacionar el método tradicional con el TDR-MT la correlación fue negativa con un valor de $\mathrm{r}$ de $-0,18$. Aunque los valores absolutos obtenidos en

Cuadro 2. Correlaciones simples de Pearson entre las variables de humedad volumétrica medido con el método tradicional y 3 TDR a 2 profundidades del suelo en el "cable 8" en Finca Esmeralda, Guácimo, Costa Rica.

\begin{tabular}{|c|c|c|c|c|}
\hline & \multicolumn{2}{|c|}{$\begin{array}{c}\text { Método Tradi. } \\
\text { (0-30 cm) }\end{array}$} & \multicolumn{2}{|c|}{$\begin{array}{c}\text { Método Tradi. } \\
\text { (30-60 cm) }\end{array}$} \\
\hline TDR-MP $(0-30 \mathrm{~cm})$ & 0,59 & $* *$ & & \\
\hline TDR $300(0-30 \mathrm{~cm})$ & 0,69 & $* *$ & & \\
\hline TDR MT $(0-30 \mathrm{~cm}) *$ & 0,63 & $* *$ & & \\
\hline TDR-MP $(30-60 \mathrm{~cm})$ & & & 0,38 & $* *$ \\
\hline TDR $300(30-60 \mathrm{~cm})$ & & & 0,47 & $* *$ \\
\hline TDR MT $(30-60 \mathrm{~cm}) *$ & & & $-0,18$ & ++ \\
\hline
\end{tabular}

* Correlaciones realizadas con un $\mathrm{N}$ de 16 datos, para todas las variables.

** Diferencias significativas a p menor a 0,01; análisis de correlación de Pearson.

++ Diferencias significativas a p menor a 0,10 ; análisis de correlación de Pearson. 
ambas profundidades y en cada tipo de aparato fueron diferentes, se mantuvo un cierto grado de proporcionalidad ya que miden la humedad del suelo bajo el mismo fundamento.

\section{Textura del suelo}

En relación con la textura, se encontraron 5 clases texturales en la capa superficial ( 0 a 30 $\mathrm{cm}$ ), de las cuales la textura franco, fue la más dominante en el área de estudio con un 44,44\% de las muestras; la franco limoso, por otro lado fue el menos frecuente con el 5,56\% de las muestras procesadas, en tanto los texturas obtenidas a $30 \mathrm{~cm}$ igualmente fueron 5 clases texturales, donde la textura Franco arcillosa fue la más dominante en el área de estudio ya que representó el $63,89 \%$, en tanto que la arcillosa fue el valor menor representativo con el 5,56\% de las muestras procesadas.

Lo encontrado en los análisis texturales concuerda con Arias et ál. (2010), quien menciona que los suelos del noroeste de la zona Atlántica de Costa Rica, presentan en sus horizontes superficiales y subsuperficiales texturas que van desde arena franca hasta franco arcillosa. En el Cuadro 3 se resumen los resultados obtenidos en el análisis textural, donde se pueden observar los valores de los porcentajes de arenas, limos, arcillas.

Cuadro 3. Parámetros estadísticos del porcentaje de arenas, limos y arcillas a 2 profundidades en el "cable 8 " en Finca Esmeralda, Guácimo, Costa Rica con un n=36.

\begin{tabular}{lccccc}
\hline Fracción (\%) & Prof. (cm) & Promedio & Máximo & Mínimo & $\begin{array}{c}\text { Desviación } \\
\text { estándar }\end{array}$ \\
\hline \multirow{2}{*}{ Arena } & 0 a 30 & 46,57 & 65,23 & 23,46 & 9,91 \\
& 30 a 60 & 37,76 & 62,39 & 21,13 & 10,03 \\
\hline \multirow{2}{*}{ Limo } & 0 a 30 & 30,48 & 60,43 & 6,6 & 9,69 \\
& 30 a 60 & 31,82 & 48,11 & 15,28 & 6,37 \\
\hline \multirow{2}{*}{ Arcillas } & 0 a 30 & 22,96 & 35,96 & 10,13 & 6,77 \\
& 30 a 60 & 30,42 & 43,02 & 10,09 & 7,97 \\
\hline
\end{tabular}

\section{Variación espacial de la humedad del suelo en el lote de estudio}

Con los datos obtenidos en el Cuadro 4, se utilizó el interpolador Kriging para representar el comportamiento de las variables de humedad en el suelo, por estar fundamentado en técnicas geoestadísticas que validan el modelo de mejor ajuste al tiempo que se unifica el criterio de tipo de interpolador para las variables de este estudio. Henríquez et ál. (2005) y Villatoro et ál. (2008), han utilizado este método, para obtener los modelos de mejor ajuste en diferentes propiedades del suelo; para ello se utilizó como base la suma de cuadrados más baja, la cual está asociada con una mejor estimación de los semivariogramas.

En relación con los resultados de la representación de la variación espacial, es importante aclarar que debido a problemas técnicos con el equipo de medición TDR-MT, no se pudieron completar las medidas de todo el área de estudio con este equipo (sólo se pudieron completar 16 de las 36 mediciones) por lo que quedó un sector importante de la finca sin las mediciones de este instrumento; ello provocó que no fuese posible completar esta fase de representación gráfica con el TDR-MT, aunque sí con el TDR300 y TDR-MP en comparación con el método 
Cuadro 4. Parámetros de los semivariogramas para algunas variables del estudio utilizando el modelo Kriging, a 2 profundidades del suelo en el "cable 8" en Finca Esmeralda, Guácimo, Costa Rica.

\begin{tabular}{lcccc}
\hline Variable & $\begin{array}{c}\text { Modelo de mejor } \\
\text { ajuste }\end{array}$ & Nugget Co & Sill Co+C & Rango Ao \\
\hline Arcilla $(0-30 \mathrm{~cm})$ & Exponencial & 0,1 & 42,42 & 50,4 \\
Arcilla $(30-60 \mathrm{~cm})$ & Esférico & 14,3 & 66,67 & 90 \\
Método tradicional $(0-30 \mathrm{~cm})$ & Gaussiano & 27,7 & 68,82 & 190,52 \\
Método tradicional $(30-60 \mathrm{~cm})$ & Esférico & 0,5 & 15,23 & 24,9 \\
TDR-MP (0-30) & Esférico & 0,05 & 11,77 & 24,9 \\
TDR-MP (30-60) & Exponencial & 4,45 & 12,015 & 87,9 \\
TDR-300 (0-30) & Esférico & 0,1 & 97,4 & 24,9 \\
TDR-300 (30-60) & Gaussiano & 2,89 & 34,02 & 6,92 \\
\hline
\end{tabular}

tradicional. Lo anterior significa que las mediciones del TDR-MT fueron excluidas de esta parte de la discusión que se refiere específicamente a la variación espacial de la humedad del suelo en el lote de estudio.

Los datos obtenidos en relación con la variación espacial de la humedad del suelo en el lote a 2 profundidades se presentan en la Figura 3. Como se aprecia en dicha figura, los datos o ámbitos de variación de la humedad representados en los mapas en forma espacial y visualmente, corresponden también a los valores presentados en el Cuadro 1, con la diferencia de que en el mapa se puede apreciar su variación espacial a través del paisaje y no solamente un único valor. Al visualizar los datos de humedad volumétrica, se confirma que los porcentajes de humedad variaron entre los TDR utilizados y el método tradicional. Esto en primera instancia podría estar relacionado con los factores antes mencionados como el tipo de sonda y la textura del suelo. Spectrum Technologies (2012) menciona por ejemplo que altos contenidos de arcilla $(>27 \%)$ y alta conductividad eléctrica (EC $>2$ $\mathrm{dS} / \mathrm{m}$ ) disminuyen la señal de alta frecuencia y afectan la lectura en el aparato TDR-300. Por otro lado el TDR-MP no presenta dificultad al trabajar en suelos arcillosos ya que está calibrado para suelos minerales, e incluso suelos de textura arenosa y arcillosos (ICT International 2012).
Pese a esta variación, con cualquiera de los métodos utilizados, se logró detectar un área del lote donde se encontró la mayor cantidad de humedad volumétrica en el suelo (marcada en color rojo en el mapa), característica que coincidió con la zona que presentó los valores de arcillas más altos a nivel superficial. A la profundidad de 30 a $60 \mathrm{~cm}$ por otro lado, se encontró una ligera coincidencia entre el método tradicional y el TDR-MP, no así entre el primero y el TDR-300. El TDR-MP presentó una elevada correspondencia entre mayores contenidos de humedad con mayores valores de arcilla.

Al realizar el análisis de correspondencia entre los métodos, se encontró a nivel superficial un área de coincidencia relativa entre los valores del porcentaje de humedad volumétrica $(\% \mathrm{Hv})$ del método tradicional y TDR-MP de $39,7 \%$, mientras que la correspondencia entre el método tradicional y el TDR-300 fue de $42,2 \%$. Por otro lado de 30 a $60 \mathrm{~cm}$ se encontró un área de coincidencia entre el método tradicional los métodos de TDR-MP y TDR-300, de 56,7\% y de 76,5\% respectivamente. Estos porcentajes de correspondencia representan las áreas donde las lecturas obtenidas por el método tradicional y los 2 aparatos TDR se traslaparon con base en una reclasificación previa con categorías bajo, medio y alto ya que los valores absolutos entre los métodos eran diferentes. 

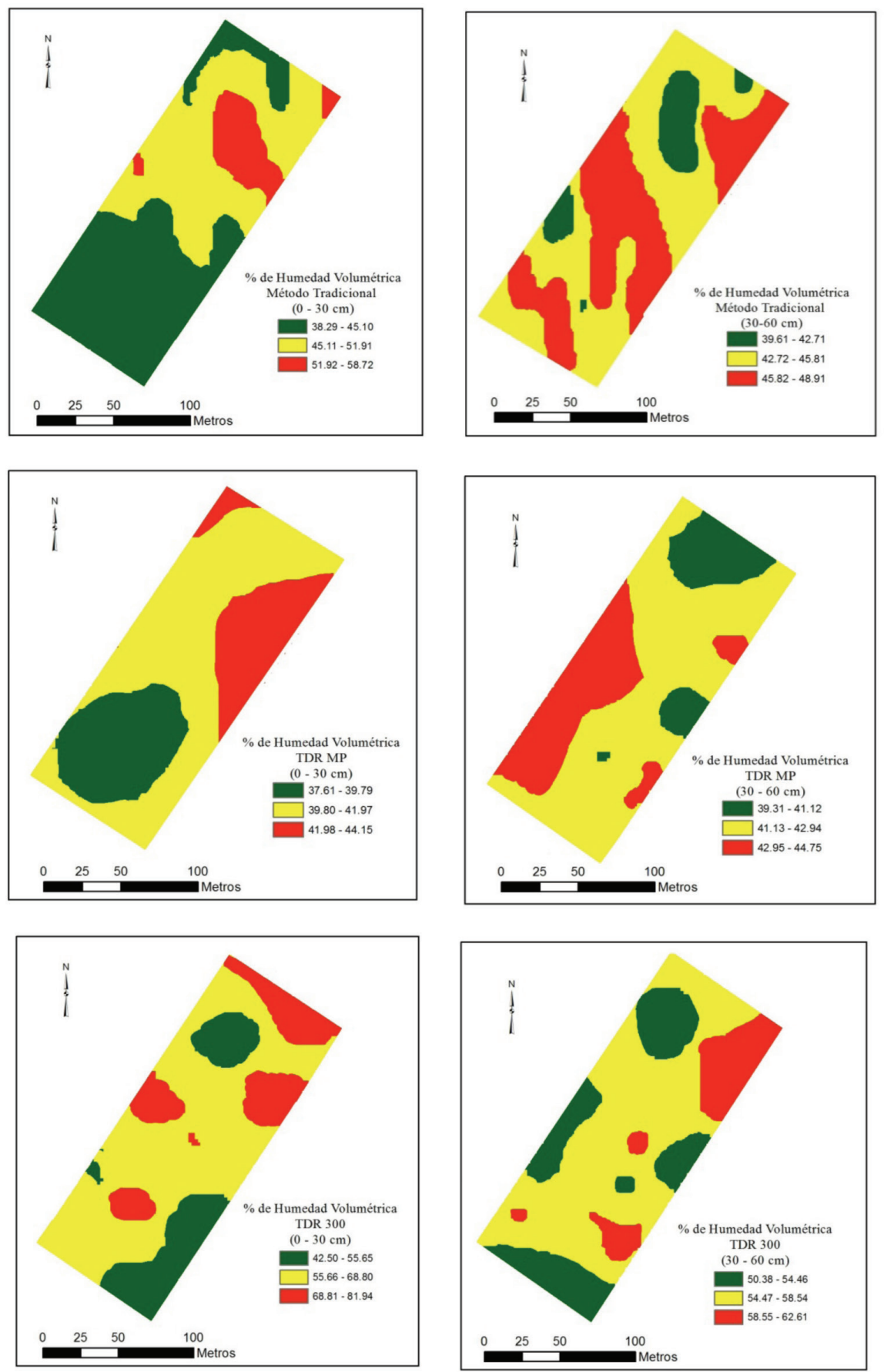

Fig. 3. Mapa de variación espacial, obtenido mediante el interpolador Co-Kriging entre los valores del \% de humedad volumétrica a nivel superficial y $30-60 \mathrm{~cm}$ y el porcentaje de arcilla como covariable. 
En general se puede decir que se encontró una buena correspondencia entre los métodos para definir zonas de alto contenido de humedad en el lote. También se halló una buena relación entre el contenido de arcilla y humedad del suelo. En relación con esto último, se sabe que las partículas de arcilla, tienen una mayor capacidad de retención y almacenamiento de agua en comparación con los limos y las arenas (Gavande 1973, Honorato 2000), esto debido principalmente a su menor tamaño, la mayor superficie específica de reacción y a la mayor cantidad de microporos por centímetro cúbico que forma dentro del suelo (Thompson y Troeh 1998). Lo anterior se confirma al correlacionar las variables de distribución espacial de la humedad del suelo determinadas en este estudio en relación con el porcentaje de arcilla, se encontró a nivel superficial, que la correlación más alta es entre los valores del método tradicional y el TDR-MT ( $\mathrm{r}$ de $0,52 \mathrm{y}$ altamente significativo), datos que se presentan en el Cuadro 5.

Cuadro 5. Correlaciones simples de Pearson entre las variables de humedad volumétrica del método tradicional y los 3 TDR vs el porcentaje de arcilla a 2 profundidades del suelo en el "cable 8" en Finca Esmeralda, Guácimo, Costa Rica.

\begin{tabular}{|c|c|c|c|c|}
\hline \multirow[b]{2}{*}{ Método Tradicional $(0-30 \mathrm{~cm})$} & \multicolumn{2}{|c|}{$\begin{array}{l}\text { Arcillas } \\
(0-30 \mathrm{~cm})\end{array}$} & \multicolumn{2}{|c|}{$\begin{array}{c}\text { Arcillas } \\
(30-60 \mathrm{~cm})\end{array}$} \\
\hline & 0,52 & $* *$ & & \\
\hline TDR-MP (0-30 cm) & 0,31 & + & & \\
\hline TDR-300 $(0-30 \mathrm{~cm})$ & 0,48 & $* *$ & & \\
\hline TDR-MT $(0-30 \mathrm{~cm}) *$ & 0,52 & $* *$ & & \\
\hline Método Tradicional $(30-60 \mathrm{~cm})$ & & & 0,34 & $* *$ \\
\hline TDR-MP (30-60 cm) & & & 0,15 & ++ \\
\hline TDR $300(30-60 \mathrm{~cm})$ & & & 0,14 & ++ \\
\hline TDR MT $(30-60 \mathrm{~cm}) *$ & & & $-0,20$ & ++ \\
\hline
\end{tabular}

* Correlaciones realizada con un $\mathrm{N}$ de 16 datos, para todas las variables.

** Diferencias significativas a p menor a 0,001; análisis de correlación de Pearson.

+ Diferencias significativas a p menor a 0,050; análisis de correlación de Pearson.

++ Diferencias significativas a p menor a 0,10; análisis de correlación de Pearson.

Además que a la profundidad de 30 a 60 $\mathrm{cm}$ se dio el mismo comportamiento, el método tradicional es el que presenta la correlación más alta $(0,34)$, en comparación a los TDRs, esto debido a que los valores de las arcillas pudieron interferir con las lecturas registradas, como se mencionó anteriormente.

Dado la alta correlación encontrada entre la humedad volumétrica y el contenido de arcillas se decidió hacer un nuevo análisis geoestadístico utilizando un co-Kriging para visualizar de una mejor manera la distribución de la humedad por medio del contenido de arcilla como covariable. En la Figura 3 se observa como mejoró la visualización de la variación de la humedad en el suelo.

\section{CONCLUSIÓN}

Con la presente investigación se puede concluir que las técnicas de reflectometría en el dominio de tiempo (TDR), mostraron ser un método eficaz para representar la variación espacial de humedad en los suelos, los valores absolutos de humedad determinada con los TDR's variaron entre sí y con respecto a la determinación de referencia que fue el método tradicional, sin embargo el análisis de correspondencias entre los métodos, mostró los mejores valores a la profundidad de 30 a $60 \mathrm{~cm}$. Pese a ello al realizar la investigación mediante el análisis de correlaciones y correspondencia se pudo determinar que el TDR-300 presentó una mejor coincidencia con 
el método tradicional, en fin los TDR's son una herramienta rápida in situ, para determinar la humedad del suelo.

\section{AGRADECIMIENTOS}

Los autores desean agradecer a la empresa Chiquita Brand por facilitar el lugar de estudio.

\section{LITERATURA CITADA}

ARIAS F., ALVARADO A., MATA R., SERRANO E., LAGUNA J. 2010. Relación entre la mineralogía de la fracción arcilla y la fertilidad en algunos suelos cultivados con banano en las llanuras aluviales del Caribe de Costa Rica. Agronomía Costarricense 34(2):223-236.

FORSTYHE W. 1985. Manual de laboratorio de física de suelos. IICA. San José, Costa Rica. 212 p.

GAVANDE S. 1973. Física de suelos: Principios y aplicaciones. LIMUSA-WILEY, S.A. $1^{\text {a }}$ reimp. México D. F. México. 351 p.

HENRÍQUEZ C., CABALCETA G. 2012. Guía práctica para el estudio introductorio de los suelos con un enfoque Agrícola. ACCS. $2^{\mathrm{a}}$ ed. San José, Costa Rica. 112 p.

HENRÍQUEZ C., KILLORN R., BERTSCH F., SANCHO F. 2005. La Geostadística en el Estudio de la Variación Espacial de la Fertilidad del Suelo Mediante el uso del Interpolador Kriging. Agronomía Costarricense 29(2):73-81.

HONORATO R. 2000. Manual de Edafología. $4^{a}$ ed. ALFAOMEGA, México. 220 p.

ICT INTERNATIONAL. 2012. MPM 160 moisture probe meter operation manual Armidale, Australia, pp. 2-10. Consultado 5 mayo de 2012. Disponible en www.ictinternational.com.au
MARTÍNEZ J., CEBALLOS A. 2001. Diseño y validación de una sonda TDR para la medición de la humedad del suelo, pp. 37-43. In: J.J. López y M. Quemada (eds.). Temas de investigación en zona no saturada. Universidad Pública de Navarra. Pamplona, España.

MUÑOZ R., RITTER A. 2005. Hidrología Agroforestal. Mundi-Prensa, Canarias, España. 348 p.

PORTA J., LÓPEZ M., ROQUERO C. 2003. Edafología para la agricultura y el medio ambiente. Ediciones Mundi-Prensa. España. 929 p.

RADULOVICH R. 2009. Método gravimétrico para determinar in situ la humedad volumétrica del suelo. Agronomía Costarricense 33(1):121-124.

SÁNCHEZ J., LAURENT J., BOHY M., AUZET A., THONY J. 1999. Evaluación de un Nuevo método de caracterización del perfil hídrico del suelo a partir de la inversión de una sola señal TDR. Estudios de la Zona No Saturada del Suelo Vol. IV. ICIA. Tenerife, España. ISBN 84-699-1258-5. pp. 33-38.

SPECTRUM TECHNOLOGIES. 2012. Manual de usuario: Fieldscout medidor de humedad de suelo TDR 300. Monterrey, México, pp. 2-12. Consultado 5 mayo de 2012. Disponible en www.kosmos.com.mx

THOMPSON L.M., TROEH F.R. 1998. Soil and Fertility. McGraw-Hill, $4^{\text {th }}$ Edition, New York, USA. 489 p.

TOPP G.C., FERRE T.P.A. 2002. Water content, In: J.H. Dane and G.C. Topp (eds.), Methods of Soil Analysis. Part 4. SSSA Book Series N ${ }^{\circ}$. 5. Soil Science Society of America, Madison WI. USA. 1692 p.

TORRÁN E. 2007. Impacto de las plantaciones de Eucalyptus grandis sobre el contenido de humedad del suelo. Tesis de maestría, Universidad Tecnológica Nacional, Concepción del Uruguay. 113 p.

VILLATORO M., HENRÍQUEZ C., SANCHO F. 2008. Comparación de los interpoladores IDW y Kriging en la variación espacial de $\mathrm{pH}, \mathrm{Ca}$, CICE y $\mathrm{P}$ del suelo. Agronomía Costarricense 32(1):95-105. 
